\title{
Turbulence Intensity Effects on a Leading-Edge Separation Bubble of Flat Plate Wing at Low-Reynolds Numbers
}

\author{
Katsuya Kajiwara, Masayuki Anyoji \\ Interdisciplinary Graduate School of Engineering Sciences, Kyushu University, Fukuoka, Japan \\ Email: kajiwara.katsuya.224@s.kyushu-u.ac.jp, anyoji.masayuki.660@m.kyushu-u.ac.jp
}

How to cite this paper: Kajiwara, K. and Anyoji, M. (2020) Turbulence Intensity Effects on a Leading-Edge Separation Bubble of Flat Plate Wing at Low-Reynolds Numbers. Journal of Flow Control, Measurement \& Visualization, 8, 188-207. https://doi.org/10.4236/jfcmv.2020.84012

Received: June 22, 2020

Accepted: August 28, 2020

Published: October 13, 2020

Copyright $\odot 2020$ by author(s) and Scientific Research Publishing Inc. This work is licensed under the Creative Commons Attribution International License (CC BY 4.0).

http://creativecommons.org/licenses/by/4.0/

\section{(c) (i) Open Access}

\begin{abstract}
In this study, we experimentally investigate the effects of mainstream turbulence intensity $\left(T_{i}\right)$ on a leading-edge separation bubble under low-Reynolds number $\left(R e_{c}\right)$ conditions range of $2.0 \times 10^{4}$ to $6.0 \times 10^{4}$. We used a flat plate to fix a separation point at the leading edge. Also, we visualized the behavior of the leading-edge separation bubble using the smoke wire technique and Particle Image Velocimetry (PIV) measurement. Furthermore, we measured the effect of $T_{i}$ on the turbulent transition process in the separated shear layer using a hot-wire anemometer. The results indicate that the bypass transition for large $T_{i}$ causes the turbulent transition, and so accelerates the reattachment of the separated shear layer. The results show that the bypass transition promotes the reattachment of the separated shear layer to maintain the leading-edge separation bubble on the upper surface even at high angles of attack, increasing the stall angle.
\end{abstract}

\section{Keywords}

Separation, Turbulence Transition, Fluid Dynamics

\section{Introduction}

As aircraft become smaller and lighter, Micro Air Vehicles (MAVs) and Nano Air Vehicles (NAVs) for collecting information at disaster sites where no one can enter and conducting terrain surveys on complex terrain were being studied [1] [2]. Since these aircraft have small wing areas and relatively low flight speed of about several tens of meters per second, they have low flight Reynolds number less than 100,000, based on the chord length. For two decades, another specific aircraft, the Mars airplane, are being developed for aerial exploration in the 
low-density Mars atmosphere [3] [4]. Also, high-performance low-Reynolds number airfoils at around the cruising Reynolds number of 20,000 have been studied [5] [6].

In such a low-Reynolds number regime, it is a fact that an early laminar separated flow undergoes turbulent transition due to the instability of the separated shear layer and reattaches, forming the so-called Laminar Separation Bubble (LSB) [7]. The change in the pressure distribution on the wing surface associated with the formation and collapse of the LSB is a dominant factor of the nonlinearity of the aerodynamic performance [8] [9]. Since the behavior of the LSB greatly depends on the airfoil shape, the angle of attack, and the Reynolds number, accurate prediction of the LSB behavior is an important issue for the aerodynamic design of high-performance low-Reynolds number airfoils. Schmitz [10] [11] has suggested the three-representative shape features with high aerodynamic performance at low-Reynolds numbers (i.e., a flat upper surface, a camber on the pressure side, and a sharpened leading edge). In particular, the sharp leading edge fixes the separation point at the leading edge, reducing the Reynolds number dependence on the behavior of the LSB and nonlinearity of the lift curve. An aerodynamic model with small Reynolds number dependence and strong lift linearity is very useful for control system design.

Another factor affecting the behavior of the LSB is the mainstream turbulence intensity $\left(T_{i}\right)$ [12] [13] [14]. Wang et al. [13] investigated the $T_{i}$ effect on both aerodynamic performance and flow field for NACA0012 airfoils under the conditions that the chord length based Reynolds number $\left(R e_{c}\right)$ ranges from $5.3 \times 10^{3}$ to $2.0 \times 10^{4}$. They found that the separation point moves downstream, and the reattachment point moves upstream in the change of $T_{i}$ from $0.6 \%$ to $6.0 \%$. Furthermore, they showed that the maximum lift coefficient $\left(C_{L}\right)$ increased by $51 \%$, and the drag coefficient $\left(C_{D}\right)$ also increased associated with the change of the flow field. However, the cause of these phenomena is mixed effects of $T_{i}$ on the turbulent transition of both the separation point and separated shear layer. It is difficult to separate each effect.

Many experiments and numerical calculations have revealed the effects of $T_{i}$ on the separated shear layer induced by the adverse pressure gradient of the attach flow [15] [16] [17]. Simoni et al. [15] experimentally investigated the effect of $T_{i}$ level on the structure and dynamics of laminar separation bubbles formed on a flat plate due to the adverse pressure gradient using time-resolved PIV measurements in the Reynolds number range from $R e_{c}=40,000$ to 90,000 for three $T_{i}$ levels ( $T_{i}=0.65 \%, 1.2 \%$, and $2.87 \%$ ). Their results show that the separation bubble decreases with increasing $R e_{c}$ and $T_{p}$ and the separation bubble is not observed, especially at the highest $R e_{c}$ of 90,000 and $T_{i}$ of $2.87 \%$. They carried out detailed a spectral analysis to identify a peak frequency of vortex shedding associated with the inviscid shear layer instability (Kelvin-Helmholtz (KH) instability) for all cases except for the highest $R e_{c}$ and $T_{i}$. In addition, $\mathrm{Li}$ and Yang [16] numerically investigated the effect of $T_{i}$ on the separation boundary layer transition induced by the adverse pressure gradient on a flat plate with a 
rounded leading edge at $T_{i}=3.0 \%$. Their results indicated that boundary layer streaks (Klebanoff distortions or modes) were formed upstream of the separation. As a result of the interaction between the distorted $\mathrm{KH}$ roll and the streak, some of the $\mathrm{KH}$ roll merges with the streak and rapidly developing into a chaotic $3 \mathrm{D}$ structure downstream. However, there is no evidence that a separated shear layer induced by an adverse pressure gradient bypasses the turbulent transition process due to $\mathrm{KH}$ instability under the increased $T_{i}$ conditions. In contrast, in order to separate whether the influence of $T_{i}$ acts on the separation point or the separated shear layer, this study focuses on the effects of $T_{i}$ on the separated shear layer which is geometrically induced by a sharp leading edge rather than an adverse pressure gradient.

To fix the separation point, the turbulence transition process of the separated shear layer using a long flat plate under low levels of $T_{i}$ less than $0.2 \%$ has been revealed that the transition process is initiated in the separated shear layer due to an inviscid instability ( $\mathrm{KH}$ instability) by many numerical calculations at the thickness based Reynolds number $\left(R e_{H}\right)$ of 3450 and 6500 [18] [19]. These initial two-dimensional (2D) spanwise vortices, called the $\mathrm{KH}$ rolls, grow downstream. Further downstream disturbed $\mathrm{KH}$ rolls transform into $\Lambda$-shape vortical structure due to helical pairing instability, leading to three-dimensionality of the flow. Those large-scale coherent structures breakdown to smaller ones, eventually leading to turbulence around the mean reattach point.

In contrast, the literature on the transition process in separation bubbles induced geometrically under elevated level of $T_{i}$ is scarce. Yang and Abdalla [20] numerically investigated the effect of $T_{i}$ of $2 \%$ at the blunt leading edge of a flat plate at $R e_{H}=6500$. They found that $2 \mathrm{D} \mathrm{KH}$ rolls were still observable, and the primary instability was shown to be the same as in the low $T_{i}$ case, whereas the transition process started earlier, resulting in a $14 \%$ reduction in the mean LSB length compared to the case of very low $T_{i}[18]$. Langari and Yang [21] numerically investigated the effects of large $T_{i}\left(T_{i}=5.6 \%\right)$ on the fluid physics around a long flat plate with a rounded leading edge at $R e_{H}=3450$. Their results suggested that those spanwise 2D KH rolls do not appear, and spanwise irregularity appears in the separated shear layer at the early stage of the separation bubble. This induces the rapid formation of the three-dimensional (3D) structures by bypassing the $2 \mathrm{D} \mathrm{KH}$ rolls stage, causing much earlier breakdown to turbulence and a $40 \%$ reduction in the length of the LSB. They concluded that a bypass transition occurs due to the bypass of the $\mathrm{KH}$ instability stage at $T_{i}=5.6 \%$. Therefore, the physical mechanism of the effect of $T_{i}$ on the turbulent transition is different between the above-mentioned adverse pressure gradient induced and geometrically induced separation shear layers.

In addition, Lee et al. [22] investigated the Reynolds number dependence of the LSB characteristics by using Large Eddy Simulation (LES) in the low $R e_{c}$ ranges from 5000 to 20,000, which corresponds to under the condition that $R e_{H}$ ranges from 250 to 1000 at $T_{i}=0 \%$. In such a very low-Reynolds number range, 
the three-dimensional coherent vortex structure of LSB changes into a spanwise two-dimensional vortex structure as the $R e_{c}$ decreases, indicating that the LSB vortex structure may also be sensitive to the $T_{i}$ at such low Reynolds numbers. The $T_{i}$ sensitivity of the LSB in such very low Reynolds numbers range is of great academic interest. However, as far as we searched, there are few studies on the effect of the $T_{i}$ on the LSB in the $R e_{c}$ less than or equal to 20,000 .

This study focuses only on the effect of $T_{i}$ on turbulence transitions of the separated shear layer in the $R e_{c}$ range of 20,000 to 60,000 using a flat plate airfoil whose separation point can be fixed at the leading edge. Also, we experimentally investigated the mechanism of the LSB and the effects of the $T_{i}$ on the aerodynamic performance with changing the angle of attack.

\section{Experimental Setup and Condition}

\subsection{Experimental Setup}

We used an aluminum flat plate of $5 \%$ thickness with a blunt leading edge as a test model. The chord length $(c)$ and span length are $80 \mathrm{~mm}$ and $180 \mathrm{~mm}$, respectively. To prevent the surface roughness from affecting the flow field, we polished the surface of the test model so that the arithmetic mean roughness is in the range of 3.2 to $6.3 \mu \mathrm{m}$. The cross-section of the flat plate is rectangular with a $4 \mathrm{~mm}$ thickness.

Figure 1 shows the experimental setup. The position of $25 \%$ of the chord length of the test model is located $450 \mathrm{~mm}$ downstream from the inlet of the test section. In all measurements, the test model is mounted perpendicular to the ground and connected to a stepping motor through a shaft for changing the angle of attack $(\alpha)$ _(Figure 2).

Aerodynamic performance is measured by a three-component force balance system (Nissho Electric, LMC-3531-5N). Each related load of the balance system is set at $5 \mathrm{~N}$ for the lift- and drag-forces and $0.5 \mathrm{Nm}$ for the momentum. The uncertain accuracy of the aerodynamic force measurement is estimated at approximately $0.2 \%$ based on a preliminary force-calibration test. The aerodynamic measurements require a slight gap between the sidewall and the test model so that no frictional force is generated between them. With reference to the study in Burns and Mueller [23], the gap of $0.9 \mathrm{~mm}$ corresponding to $0.5 \%$ of the span length is provided.

The mainstream turbulence and the unsteady flow velocity distribution on the model surface are measured using an I-type hot-wire anemometer, and the isotropy of turbulence is measured using X-type hot-wire anemometers. The material of the sensing part is tungsten with a diameter of $5 \mu \mathrm{m}$ and a length of 1 $\mathrm{mm}$. The hot-wire anemometer is connected to the Constant-Temperature Anemometer (CTA) with a $5 \mathrm{kHz}$ low-pass filter. The sampling rate is $20 \mathrm{kHz}$, and 400,000 points are measured. The horizontal movement of the hot-wire probe is performed using a motor driven traverse device. The measurement points are changed at intervals of $x / c=0.05$ from $x / c=0.05$ to 0.40 . 


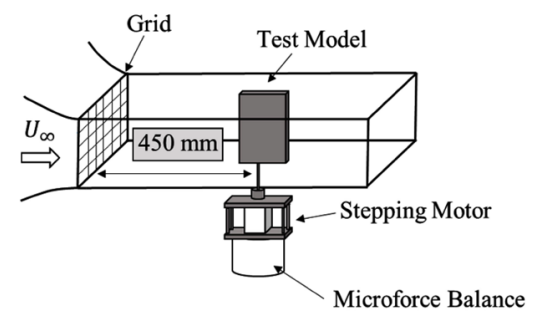

Figure 1. Schematic of force measurement.

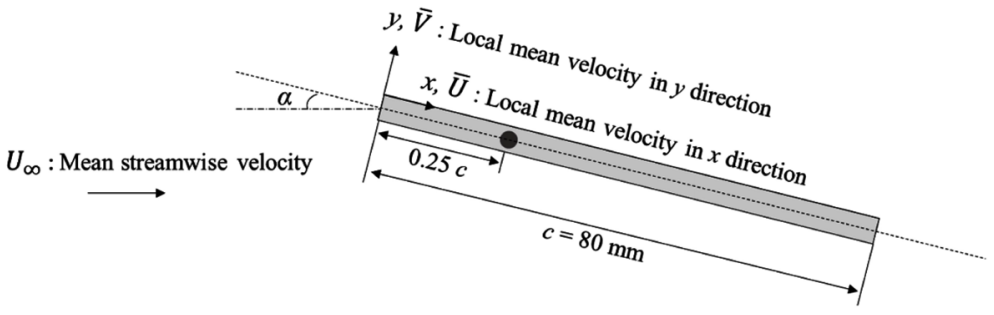

Figure 2. Test model and definition of the coordinate system.

The flow field around the test model is visualized by the smoke wire technique and PIV measurement with the same model setup as in the aerodynamic measurements. For the smoke wire measurement, two nichrome wires with $0.08 \mathrm{~mm}$ diameter are horizontally set up at the inlet of the test section for the smoke wire visualization, and liquid paraffin is used as the working substance. For the PIV measurement, evaporated vegetable oil particles with approximately $10 \mu \mathrm{m}$ dimeter are used as tracer particles. A 5W PIV-laser (COHERENT VERDI G5) is used as the light source. We took some time-series images using a high-speed video camera (Photron, AX2000) with a zoom lens (Nikon, Ai AF Zoom-Nikkor $24-85 \mathrm{~mm} \mathrm{f} / 2.8$ - $4 \mathrm{D} \mathrm{IF})$.

\subsection{Turbulence Grid and Flow Quality}

We performed experiments using an open-circuit low-speed wind tunnel at Kyushu University. One honeycomb and four screens are present in the settling chamber to reduce the turbulence level in the test section. The rectangular cross-section of the test section is $180 \mathrm{~mm} \times 360 \mathrm{~mm}$. The test section is covered on every side with an acrylic sidewall. Mainstream turbulence intensity $\left(T_{i}\right)$ in the test section is defined by:

$$
T_{i}=\frac{u_{r m s}^{\prime}}{U_{\infty}} .
$$

where $U_{\infty}$ indicates the mean value of the instantaneous streamwise velocity which is defined as a straight flow along the side walls without any deflection angle, and $u_{r m s}^{\prime}$ is Root Mean Square (RMS) value of the fluctuating component of the instantaneous streamwise velocity.

We changed the $T_{i}$ using turbulence grid (see Figure 3 ). The grid is made of stainless-steel wire with a circular cross-section. For selecting the grid configuration, a solidity factor $\left(S_{F}\right)$ of the grid expressed by Equation (2) is used as a parameter governing the attenuation of the turbulence [24]. 


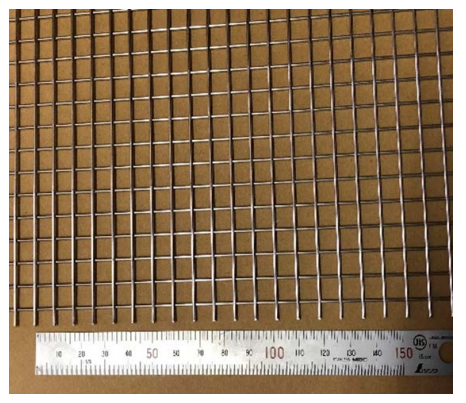

Figure 3. Turbulence grids.

$$
S_{F}=\frac{M^{2}-(M-d)^{2}}{M^{2}} .
$$

where $d$ is the wire diameter of the turbulence grid, and $M$ is the mesh size. For estimation of the $T_{p}$ the following Equation (3) indicating the turbulent energy decay is used [24]:

$$
\frac{U_{\infty}}{u_{r m s}^{\prime}}=\sqrt{\left(335-570 S_{F}\right)\left(\frac{x^{\prime}}{M}-15\right)} .
$$

where $x^{\prime}$ is the distance downstream from the grid. The grid has $d=1.2 \mathrm{~mm}$, $M=8.46 \mathrm{~mm}$. Without a turbulence grid, the $T_{i}$ of the main flow is $0.20 \%$, whereas theoretical $T_{i}$ values of the grid estimated by Equation (3) is $1.19 \%$.

In comparison with the estimated values above, the measured flow characteristics are tabulated in Table 1 . The measured $T_{i}$ of the grid is $1.26 \%$ and almost agree with the predicted value. $u_{r m s}^{\prime} / v_{r m s}^{\prime}$ is less than 1.3 , showing a quasi-isotropic mainstream turbulence for the both $T_{i}$ levels, where $v_{r m s}^{\prime}$ is RMS value of the fluctuating component of the lateral instantaneous velocity. Based on the Taylor hypothesis, the streamwise integral length scales $L_{u}$ can be estimated by Equation (4):

$$
L_{u}=U_{\infty} \int_{0}^{\tau_{0}} R_{u}(\tau) d \tau
$$

where $R_{u}$ is the autocorrelation functions of single point temporal fluctuating streamwise velocity, $\tau$ is the time difference, and $\tau_{0}$ is equal to the first zero. $L_{u}$ at $T_{i}=1.26 \%$ corresponds to $0.074 \mathrm{C}$.

Figure 4 shows the power spectrum of streamwise velocity in the mainstream with and without grid. Compared with the presence or absence of the turbulence grid, the magnitude of the power spectrum increases by the turbulence grid, but no significant peak is found in any of the cases. This indicates that the airfoil performance is not affected by fluctuations at a specific frequency.

\subsection{Experimental Conditions}

In this study, the experiments were conducted under two turbulence intensity conditions: $T_{i}=0.20 \%$ and $T_{i}=1.26 \%$. In addition to these conditions, aerodynamic measurements were performed supplementally at $T_{i}=1.06 \%$. The aerodynamic measurement was conducted at $R e_{c}=20,000,40,000$ and 60,000. Each 


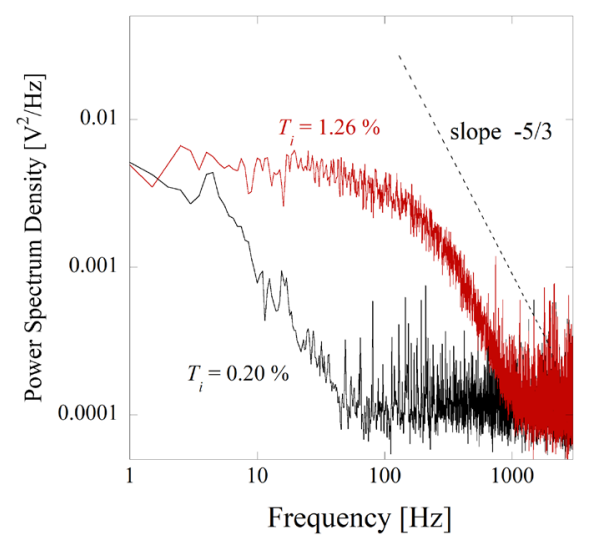

Figure 4. Power spectrum of free-stream velocity for different $T_{i}$.

Table 1. Characteristics of mainstream flow.

\begin{tabular}{cccccc}
\hline Grid & Predicted $T_{i}[\%]$ & Measured $T_{i}[\%]$ & $u_{r m s}^{\prime} / v_{r m s}^{\prime}$ & $L_{u} / c$ \\
\hline No & & 0.20 & 1.12 & \\
Yes & 1.19 & 1.26 & 1.26 & 0.074 \\
\hline
\end{tabular}

wind speed corresponds to $4.0,8.0$ and $12.0 \mathrm{~m} / \mathrm{s}$, respectively. The angle of attack $(\alpha)$ was changed from -10 to $15 \mathrm{deg}$. Since the flat plate is a symmetrical airfoil, the aerodynamic performance and fluid phenomenon should be symmetrical with positive and negative angles of attack. Therefore, although the aerodynamic measurement is performed up to the negative angles of attack, the discussion will be based on the data at positive angles of attack. In common with PIV and smoke wire measurements, the flow visualization was conducted only at $R e_{c}=20,000$ in the range from $\alpha=0$ to $12 \mathrm{deg}$. The camera resolution and frame rate were $1024 \times 880$ pixels and 5000 frames per second, respectively. The spatial resolution was $0.15 \mathrm{~mm} / \mathrm{pixel}$, which is calculated from the fact that a chord length of $80 \mathrm{~mm}$ corresponds to approximately 530 pixels on the obtained image. The recursive correlation method was used as the calculation algorithm for PIV analysis. Noted that the flow visualizations by PIV and smoke wire technique were not performed because of the limitation of PIV laser intensity and camera specification more than $R e_{c}=20,000$.

\section{Results and Discussion}

\subsection{Difference of Flow Fields with and without Turbulence Grid}

Figure 5 shows the instantaneous flow fields visualized by the smoke wire measurements. We note it is not because of fluid inside the separated shear layer that the smoke wire visualization is effective for observing the motion of the separated shear layer from the leading edge. Although Figure 5 clearly shows the separation location is fixed at the leading edge in all cases, it is still hard to determine the presence or location of the reattachment. We will discuss the details of the reattachment in the averaged flow field by the PIV measurement described later. 


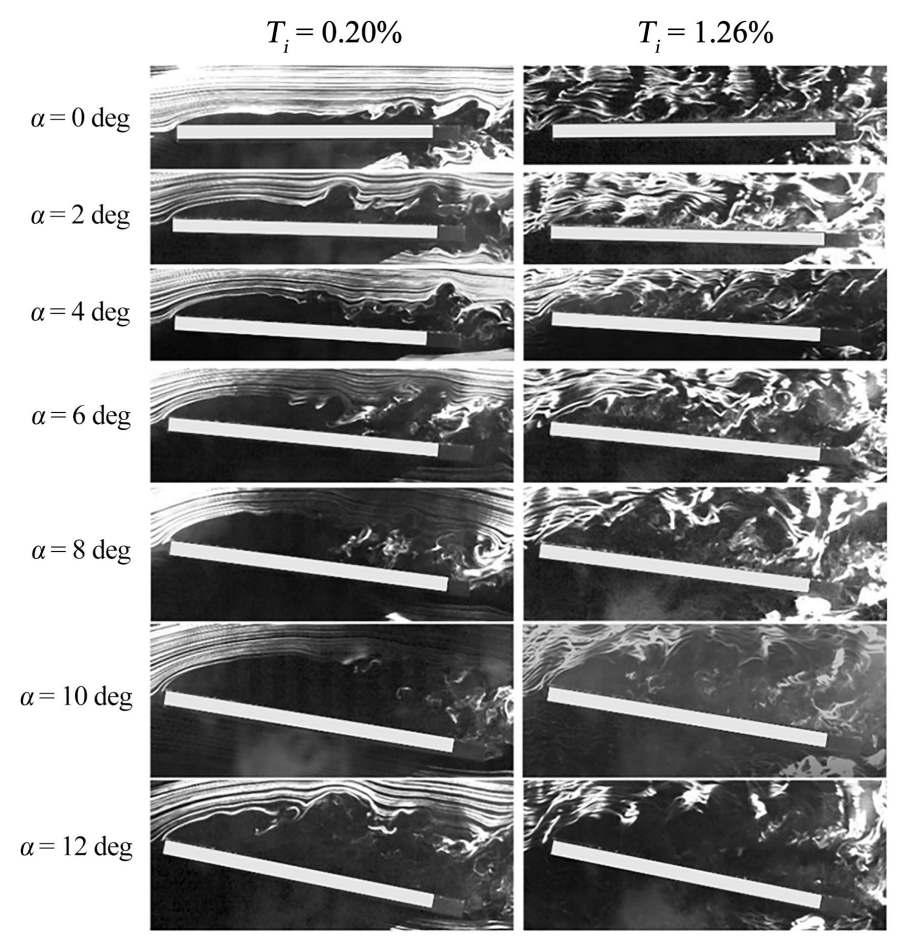

Figure 5. Instantaneous flow fields visualized by smoke-wire measurement at $R e_{c}=20,000$.

As a general and distinguishing flow field [21] [22], at $\alpha=0^{\circ}$ and $2^{\circ}$ in $T_{i}=$ $0.20 \%$, the $2 \mathrm{D}$ and stable separated shear layer gradually becomes unstable in the downstream direction, and eventually, the $2 \mathrm{D}$ coherent vortex is formed by rolling up the separated shear layer. In contrast, for $T_{i}=1.26 \%$, the shear layer becomes unstable immediately after separation regardless of the angle of attack in conjunction with the mainstream turbulence, and turbulent vortices are advected on the surface from the upstream.

Figure 6 compares the averaged velocity distribution by the PIV measurements of $T_{i}=0.20 \%$ and $T_{i}=1.26 \%$ at $R e_{c}=20,000$. The color contour indicates the flow velocity ratio of the local mean velocity $(\bar{U})$ which is defined as the time mean velocity in the $x$ direction at each measurement point normalized by the mean streamwise velocity $\left(U_{\infty}\right)$. As in numerous previous studies [22] [25] [26], the flow separates at the leading edge and then reattaches to form a leading-edge LSB. The reattachment location defined as the point where the flow direction switches from the reverse direction to the forward direction is denoted by $R$ in Figure 6. The reattachment location at $\alpha=0^{\circ}$ for $T_{i}=0.20 \%$ is $x / c=$ 0.35 , which is close to the LES results under $T_{i}=0 \%$ by Lee et al. [22]. Therefore, the slight difference between $T_{i}=0 \%$ and $0.20 \%$ has little effect on the flow field. The LSBs form in both cases until $\alpha=8^{\circ}$, and the reattachment location gradually moves downstream as the angle of attack increases, indicating the characteristics of the so-called long bubble [27]. However, for $T_{i}=1.26 \%$, the reattachment location moves upstream, and the length of LSB becomes shorter compared to $T_{i}=0.20 \%$. In particular, at $\alpha=0^{\circ}$, the LSB length is reduced by $54 \%$ 
compared to $T_{i}=0.20 \%$. At a high angle of attack of $\alpha=10^{\circ}$, in $T_{i}=0.20 \%$, the reattachment location passes through the trailing edge, and the LSB expands into the wake. In contrast, in $T_{i}=1.26 \%$, the LSB still exists by the premature reattachment on the upper surface. It can be said that the increase in $T_{i}$ promotes reattachment, and thus works to keep the LSB on the upper surface as high angles of attack as possible. At $\alpha=12^{\circ}$ (Figure $6(\mathrm{~g})$ and Figure $6(\mathrm{n})$ ), no reattachment is observed in both cases.

Figure 7 compares the Turbulent Kinetic Energy (TKE) fields of $T_{i}=0.20 \%$ and $T_{i}=1.26 \%$ at $\alpha=0^{\circ}$. According to the LES results by Lee et al. [22], 2D coherent vortices from the separated shear layer break down to the $3 \mathrm{D}$ turbulent structures. Also, the TKE becomes large at the location where these $3 \mathrm{D}$ turbulent structures take place in association with this breakdown. However, the TKE is very small in the separated shear layer with the $2 \mathrm{D}$ coherent structure. Figure 7 shows that the 3D turbulent structures occur at around $x / c=0.30$ and $x / c=0.15$ in $T_{i}=0.20 \%$ and $T_{i}=1.26 \%$, respectively. In this paper, we define the phenomena in which such a $3 \mathrm{D}$ turbulence structure takes place, and the TKE increases as turbulent transition of the separated shear layer and is denoted by $T$ in Figure 7. This clearly indicates that an increase of the mainstream turbulence intensity accelerates the collapse of the $2 \mathrm{D}$ separated shear layer and promotes the turbulent transition.

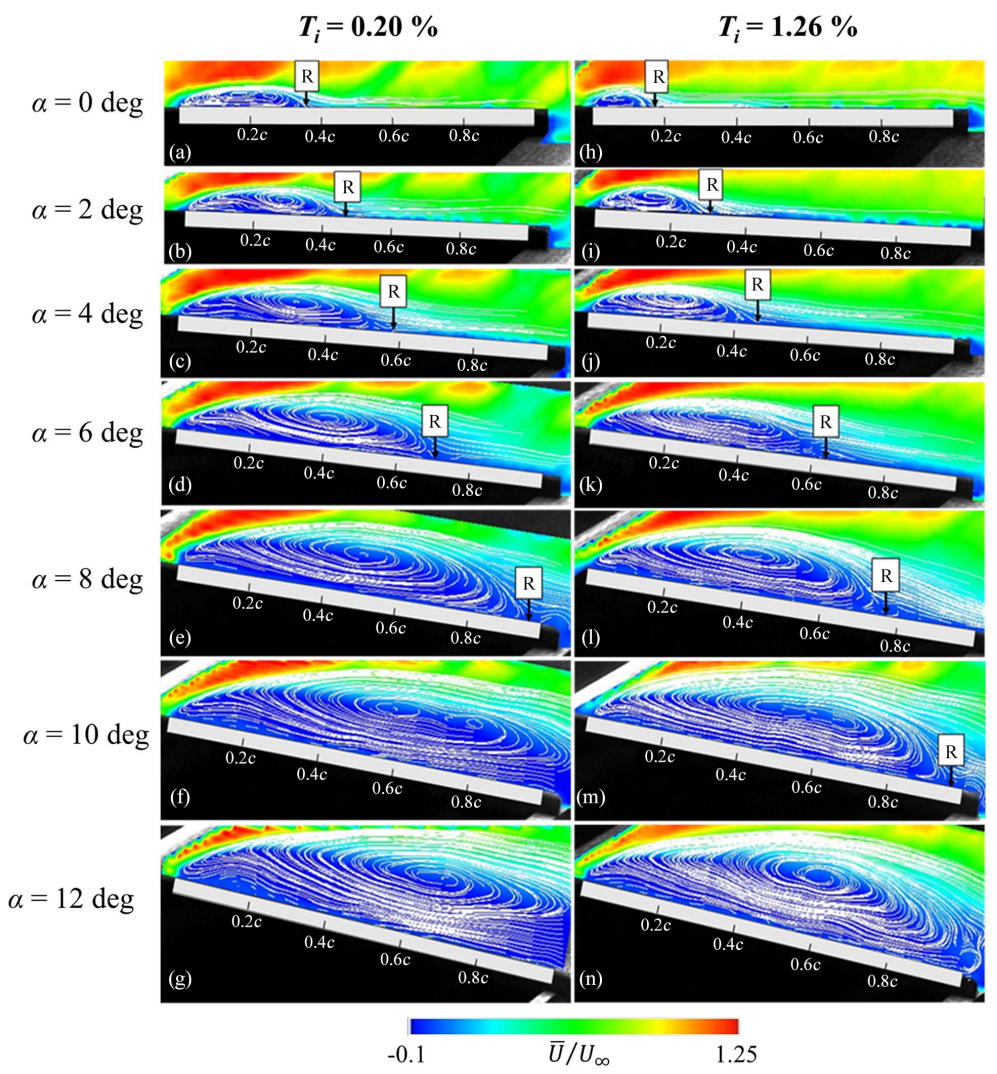

Figure 6. Local mean velocity distribution and stream lines on suction side at $R e_{c}=20,000$ by PIV measurement. 


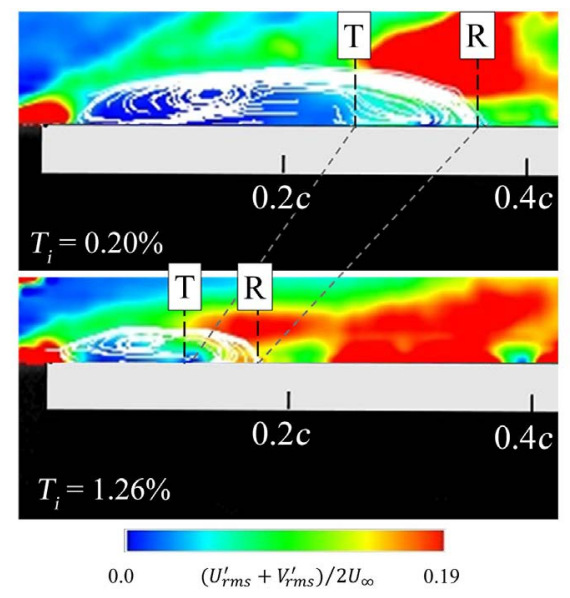

Figure 7. T.K.E fields near the leading edge of $\alpha=0$ deg at $R e_{c}=20,000$.

To investigate the effect of the $T_{i}$ on the turbulence transition process of the separated shear layer, the velocity field around the flat plate is measured using an I-type hot-wire anemometer. We note that the hot-wire measurements have high accuracy in the attached laminar and turbulent boundary layers, and in the separated shear layer outside of the recirculation region. However, in the recirculating flow regions inside the LSB, higher measurement uncertainty results from low-speed calibration error, hot-wire voltage rectification error, error due to enhanced conductive heat loss near the model surface, and error due to relatively high mean vertical flow speed [28] [29] [30]. In order to confirm the validity of the error between PIV measurement and hot-wire measurement, the comparison of both data is shown in Figure 8. The velocity in the PIV measurement indicates the local mean velocity along the flat plate $(\bar{U})$, whereas the velocity in the hot-wire measurement shows the local mean velocity including the $y$ component $\left(\sqrt{\bar{U}^{2}+\bar{V}^{2}}\right)$ because the velocity components cannot be decomposed in the I-type hot-wire measurement. However, by comparing $\bar{U}$ and $\sqrt{\bar{U}^{2}+\bar{V}^{2}}$ in the PIV measurement, it was preliminarily confirmed that there was almost no difference between $\bar{U}$ and $\sqrt{\bar{U}^{2}+\bar{V}^{2}}$ except for the backflow region, especially at $a=0 \mathrm{deg}$. Therefore, the flow velocity in the hot-wire measurement can be treated as the local mean velocity in the $x$ direction $(\bar{U})$, especially near the separated shear layer. Generally, I-type hot-wire cannot identify backflow inside the LSB. Indeed, Figure 8 reveals that the PIV results capture the backflow inside the separated shear layer that was not observed by the hot-wire measurement. Furthermore, although the velocities in the uniform flow region agree well with each other, there is a slight difference in the velocity gradient in the separated shear layer. We note that this velocity difference in the separated shear layer does not affect the conclusion of the $\mathrm{KH}$ instability criterion discussed later. In addition to the above, in consideration of the frame rate of the high-speed video camera and the emission intensity of the light source in the PIV measurement, the result of the hot-wire measurement, which is considered to have higher accuracy, was used for the calculation of $K h$. 


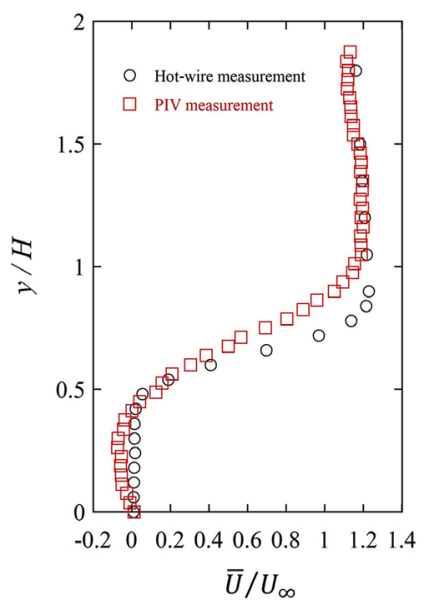

Figure 8. Comparison of the local mean velocity distribution between PIV measurement and hot-wire measurement at $x / c=0.20$ for $T_{i}=0.20 \%$.

Figure 9 shows the local mean velocity profiles in the $x$ direction around the leading edge at $\alpha=0^{\circ}$ and $R e_{c}=20,000$. The vertical axis indicates the height from the wing surface $(y)$ normalized by the flat plate thickness $(H)$. In $T_{i}=$ $0.20 \%$, a change in the velocity profile is observed at $x / c=0.25$, and the velocity gradient becomes larger from $x / c=0.30$. This is consistent with the turbulent transition described above. In contrast, in $T_{i}=1.26 \%$, the velocity profile starts to change at $x / \mathcal{c}=0.10$ attributed to the early turbulent transition. Also, the difference in the velocity gradient becomes more remarkable downstream of $x / c=$ 0.15 compared to $T_{i}=0.20 \%$. Figure 10 shows the RMS of local mean velocity fluctuations profiles. In both $T_{i}=0.20 \%$ and $T_{i}=1.26 \%$, the flow fluctuations are activated by the turbulence transition of the separated shear layer.

Figure 11 indicates the frequency characteristics of the velocity fluctuation at the maximum RMS points at $x / c=0.05$ in Figure 10. The frequency is denoted by $f$. The influence of the turbulence intensity appears in the presence or absence of the prominent peak in the spectrum. Figure 11(a) shows that in $T_{i}=0.20 \%$, as in numerous previous studies [31] [32] [33], a characteristic peak appears in the spectrum at $f=0.174 U_{\infty} / H$. According to Chandrasekhar [34], for incompressible free shear layers, the criterion for $\mathrm{KH}$ instability is $0<K h<1.2788$, where $K$ is the wavenumber and $h$ is the thickness of the separated shear layer. For the calculation method of $K$ and $h$, we referred to the past study by Langari and Yang [21]. The wave speed, $C$ is equal to the velocity at the critical layer, i.e., the streamwise velocity at the inflection point. Based on the velocity distribution at $x / c=0.05$ in Figure 9, $C$ is estimated to be $0.802 U_{\infty}$, resulting in $K=$ $2 \pi f / C=1.366 / H$. The shear layer thickness, $h$ is defined as the length from the flat plate surface to the point where a uniform flow begins to change in the velocity distribution in Figure $9(x / c=0.05)$. The shear layer thickness is estimated to be approximately $0.60 \mathrm{H}$ and hence $K h=0.82$. This meets the $\mathrm{KH}$ instability criterion $(0<K h<1.2788)$. This suggests that the turbulent transition in the separated shear layer is due to $\mathrm{KH}$ instability in $T_{i}=0.20 \%$. On the other hand, 
Figure 11(b) shows that in $T_{i}=1.26 \%$, the characteristic frequency peak did not appear in the spectrum. On the other hand, Figure 11(b) shows that in $T_{i}=$ $1.26 \%$, the characteristic frequency peak did not appear in the spectrum. Instead of the frequency peak, we used the average characteristic frequency $\bar{f}=\sum(P f) / \sum P$, where $P$ is the power spectrum density [21]. Consequently, $\bar{f}$ is estimated to be $0.262 U_{\infty} / H$. When calculating the wave velocity and the shear layer thickness as in $T_{i}=0.20 \%, C=0.599 U_{\infty}$ and $h=0.66 H$ are obtained, resulting in $K h=1.81$. This does not meet the $\mathrm{KH}$ instability criterion. Therefore, as Langari and Yang [21] suggested, the transition process due to $\mathrm{KH}$ instability bypasses and induces a bypass transition in $T_{i}=1.26 \%$ with increased

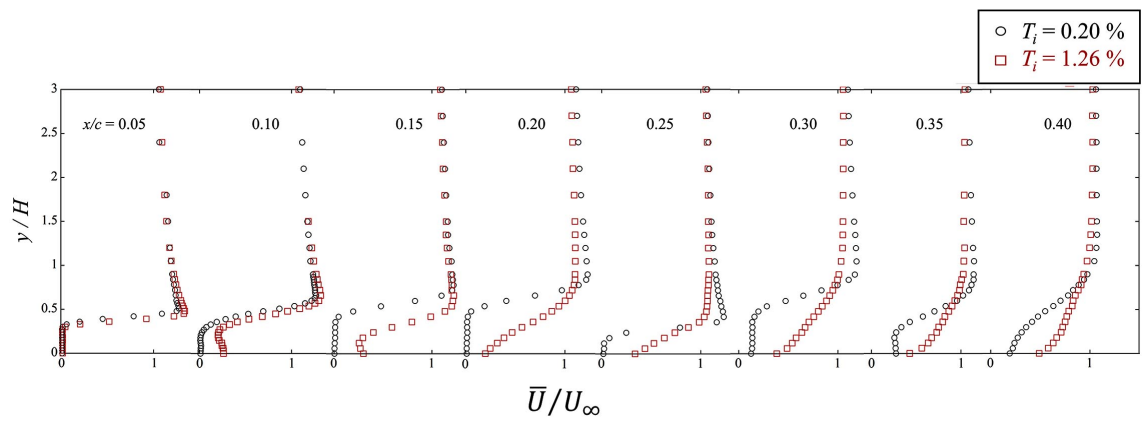

Figure 9. Local mean velocity profiles around leading edge at $\alpha=0$ deg for $R e_{c}=20,000$.

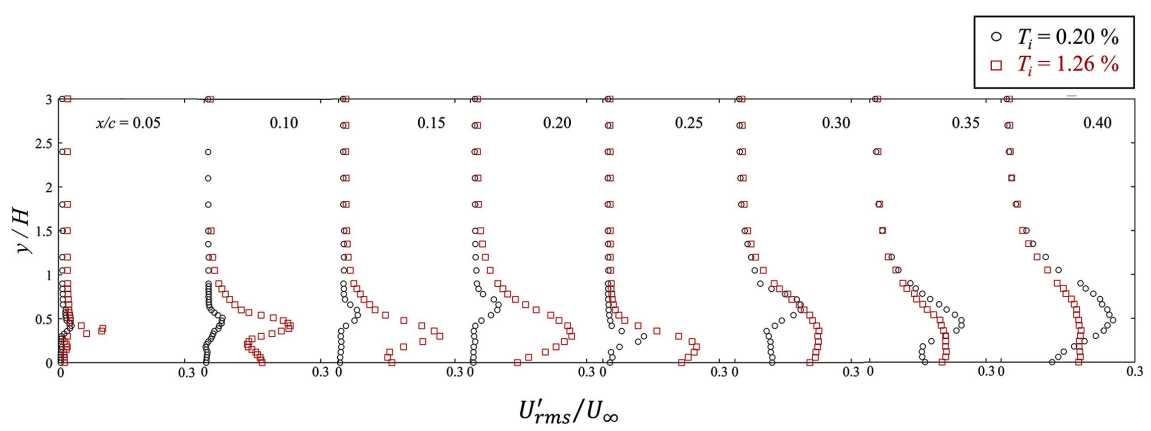

Figure 10. RMS of local velocity fluctuations profiles at $\alpha=0$ deg for $R_{c}=20,000$.

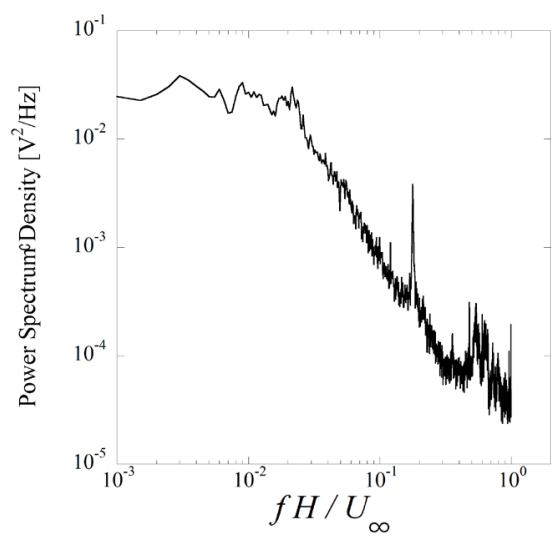

(a) $T_{i}=0.20 \%$

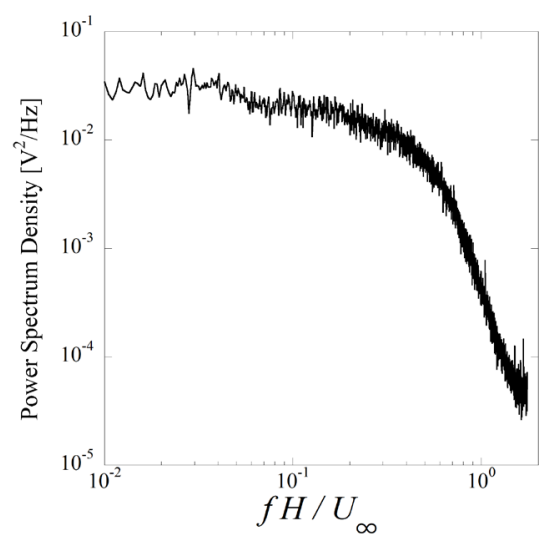

(b) $T_{i}=1.26 \%$

Figure 11. Boundary layer frequency characteristics. 
$T_{i}$. From the above, the increase in $T_{i}$ promotes turbulent transition due to a bypass transition and moves the reattachment point upstream.

\subsection{Aerodynamic Performance}

Figure 12 shows the effects of changes in the $T_{i}$ on the lift, drag, and lift-to-drag $(L / D)$ ratio characteristics of the flat plate at $R e_{c}=20,000$. Firstly, it shows that there is almost no difference in lift, drag, and $L / D$ between $T_{i}=1.06 \%$ and $T_{i}=$ $1.26 \%$, and the important factor for aerodynamic change is not the slight difference in $T_{i}$ depending on the type of turbulence grid, but the increase in turbulence intensity of $0.8 \%$ or more due to the insertion of the turbulence grid. Although a slight difference in $L / D$ due to a small difference in drag is observed at low angles of attack from $0^{\circ}$ to $9^{\circ}$, there is almost no change in aerodynamic performance regardless of whether it is with or without the turbulence grid. However, as described above, the pressure distribution on the upper surface should change since the length of the LSB is different even at low angles of attack. Therefore, we initially expected aerodynamic changes. Lee et al. [22] shows the pressure distribution at $\alpha=0^{\circ}$ and $R e_{c}=20,000$ by LES. This is shown in Figure 13. The large negative pressure region including an unchanging area corresponding to the dead water region is formed inside the LSB. Besides, the formation of the 3D hairpin vortex at around $x / c=0.20$ due to a collapse of the $2 \mathrm{D}$ vortex promotes mixing and restores the pressure on the surface. The typical features of the pressure distribution of the LSB have been explained by Tani [35] and O' Meara and Mueller [36]. The pressure distribution inside the LSB has the following three major features: 1) Pressure in a dead water region after separation is kept constant, 2) A sudden pressure increase occurs after transition, 3) A pressure recovery rate sharply decreases at a reattachment point. Furthermore, a mathematical estimation for calculating the locations of separation, turbulent transition, and reattachment based on the pressure distribution has been proposed by Gerakopulos et al. [37]. Based on these characteristics, the pressure distribution in $T_{i}=1.26 \%$ was predicted and shown in Figure 13 . The reattachment points and turbulent transition points was determined from Figure 7. From Figure 12(a), we deduced that the reason why the lift slope does not change is that the integral of the negative pressure inside the LSB does not change even when the length of LSB is shortened by increasing $T_{i}$. To verify this deduction, the pitching moment characteristics are investigated instead of the pressure distribution, which is difficult to measure using static pressure tubes because of the small thickness of the test model. Figure 14 shows the comparison of the pitching moment coefficients between $T_{i}=0.20 \%$ and $T_{i}=1.26 \%$, where the moment center is $25 \%$ of the chord length. The pitching moment coefficients increase as $T_{i}$ increases, at low angles of attack range of $1^{\circ}$ to $10^{\circ}$. This indicates that the negative pressure inside the LSB upstream of $x / c=0.25 c$ associated with the shorter LSB in $T_{i}=1.26 \%$, and the positive moment coefficient increases. This is shown in Figure 13. Besides, we assumed that there is a trade-off between increasing and decreasing the pressure distribution in $T_{i}=$ 
$0.20 \%$ and $T_{i}=1.26 \%$, which results in almost no difference in the aerodynamic coefficients at low angles of attack. We note that the flat plate is a symmetrical wing, so $\alpha=0^{\circ}$ and $C_{m 0.25 c}=0$, in all cases.

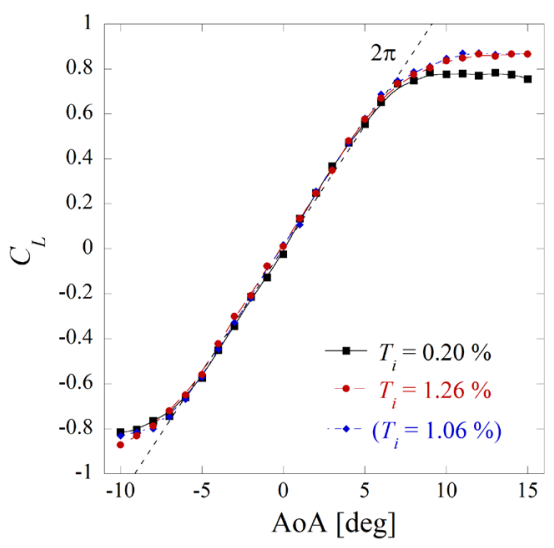

(a) Lift curve

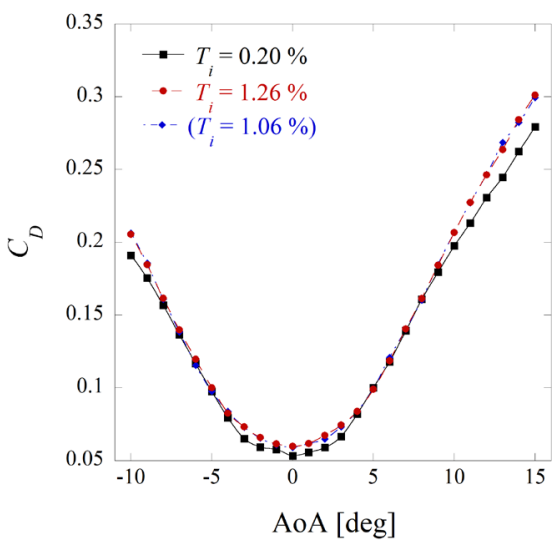

(b) Drag curve

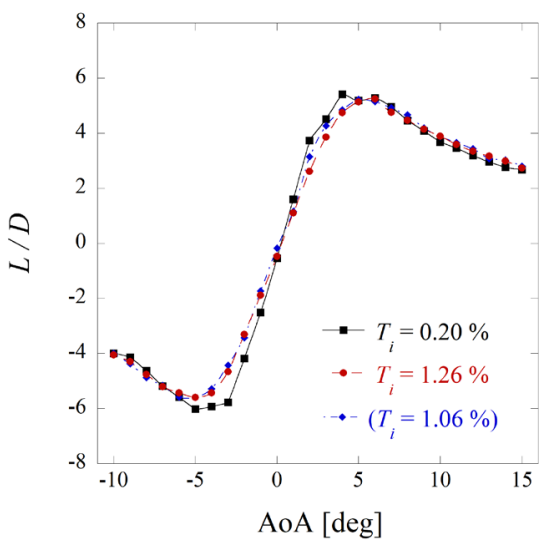

(c) Lift/Drag

Figure 12. Aerodynamic performance of flat plate at $R e_{c}=20,000$.

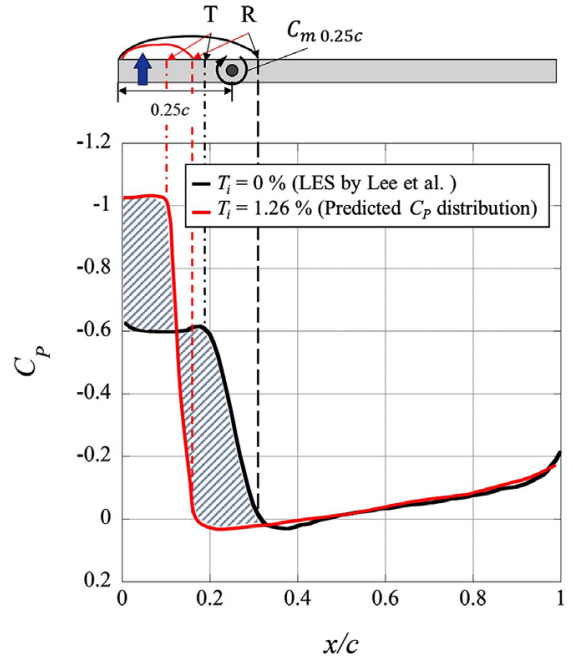

Figure 13. Predicted pressure distribution on the upper surface at $\alpha=0$ deg for $R e_{c}=20,000$ compared to LES by Lee et al. [22]. 


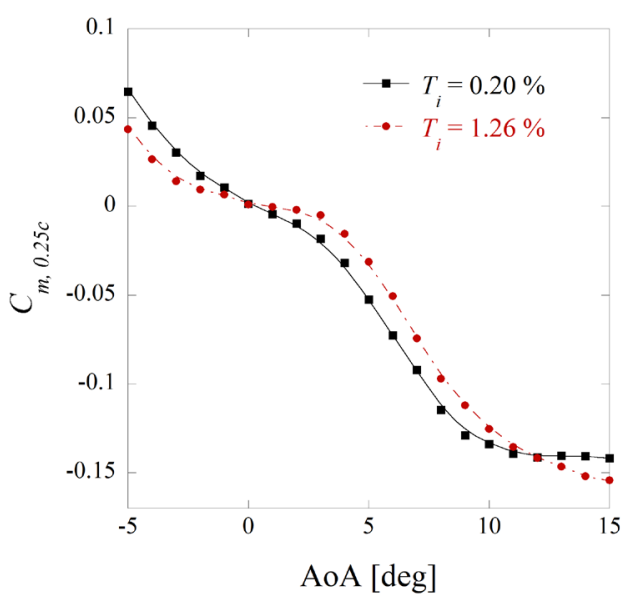

Figure 14. Pitching moment coefficient at $R e_{c}=20,000$.

For the 2D potential flow, the lift-curve slope is nearly linear with little difference in the lift coefficients in all cases and is very close to $d C_{L} / d \alpha=2 \pi$. In contrast, the remarkable difference that the lift and drag increase is observed at more than $\alpha=10^{\circ}$. This is majorly because of the difference in the flow field as shown in Figure 5 and Figure 6. The LSB keeps existing on the upper surface up to about $\alpha=10^{\circ}$. Therefore, both the lift and drag continue to increase as the angle of attack increases. However, in $T_{i}=0.20 \%$ around $\alpha=10^{\circ}$ where the flow completely separates at the leading edge without reattachment, the lift coefficients are almost at the same level since the negative pressure is maintained by the breakdown of the coherent leading-edge vortex [38]. In these results, there is no change in the $L / D$ ratio at high angles of attack because they both increase in lift and drag. The characteristic that the aerodynamic change occurs only at high angles of attack due to the increase in $T_{i}$ is consistent with the results of NACA0012 airfoil by Wang et al. [13].

Figure 15 shows the effect of $T_{i}$ on the lift coefficients at $R e_{c}=40,000$ and $R e_{c}$ $=60,000$. Although the lift slope slightly increases compared to the results at $R e_{c}$ $=20,000$ shown in Figure 15(a), the Reynolds number effect on the aerodynamic performance is almost negligible at high angles of attack of more than $10^{\circ}$. In contrast, the difference in the lift coefficients at high angles of attack gradually decreases as the Reynolds number increases. Since the increase in Reynolds number has almost the same fluid effect as the increase in $T_{i}$, these is almost no aerodynamic effect due to the $T_{i}$ of approximately $1 \%$ when $R e_{c}$ is more than 60,000 .

\section{Conclusions}

In this study, we evaluated the effects of the mainstream turbulence intensity $\left(T_{i}\right)$ on the turbulence transition of the separated shear layer and the aerodynamic characteristics of the flat plate at low $R e_{c}$ range of $R e_{c}=20,000$ to 60,000 . Qualitative flow visualization by a smoke wire method and quantitative flow analysis by a PIV measurement is carried out. The unsteadiness of the flow on the upper surface is investigated by a hot-wire anemometer. 


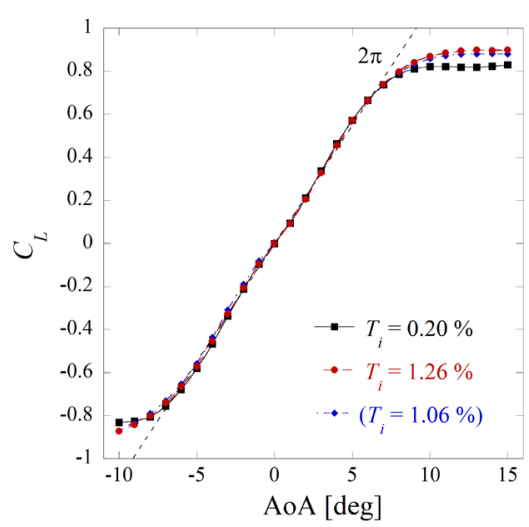

(a) $R e_{c}=40,000$

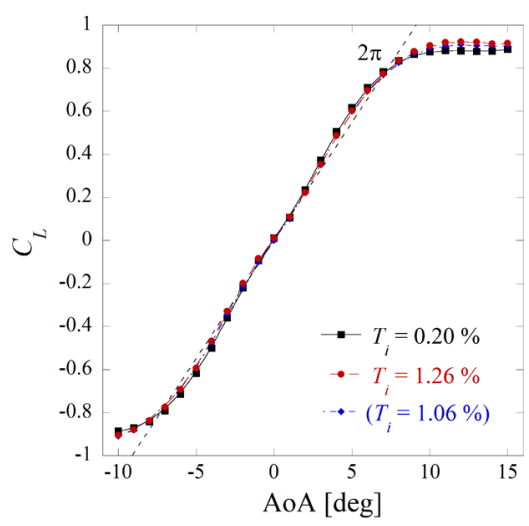

(b) $R e_{c}=60,000$

Figure 15. Comparison of lift curves of flat plate at $R e_{c}=40,000$ and $R e_{c}=60,000$.

The results of smoke wire visualization indicate that an increase in $T_{i}$ causes an unstable separated shear layer immediately after separation and the turbulent vortex shedding. Based on the Chandrasekhar's criterion, the separated shear layer undergoes the turbulent transition because of the $\mathrm{KH}$ instability for low $T_{i}$. However, the increase in $T_{i}$ promotes the turbulent transition of the separated shear layer due to the bypass transition even at $R e_{c}=20,000$, and consequently the reattachment location moves more in the upstream. The effect of $T_{i}$ on the length of LSB becomes more pronounced at lower $R e_{c}$. This early reattachment delays the collapse of the LSB since the LSB continues to exist on the upper surface even at high angles of attack. Thus, both the lift and drag increase at high angles of attack. Consequently, the lift-to-drag ratio hardly changes due to the trade-off. Even when the $T_{i}$ is changed from $1.06 \%$ to $1.26 \%$, there was almost no change in the aerodynamic performance. As the Reynolds number increases, the effects of $T_{i}$ increase on the aerodynamic performance gradually subside.

\section{Acknowledgements}

We would like to express our sincere thanks to A. Yakeno of Tohoku University for valuable discussion of this research. We would also like to thank H. Ono, who is a current graduate student at Kyushu University, for his support to the PIV measurement.

\section{Conflicts of Interest}

The authors declare no conflicts of interest regarding the publication of this paper.

\section{References}

[1] Mueller, T.J. (1999) Aerodynamic Measurements at Low Reynolds Numbers for Fixed Wing Micro-Air Vehicles. DTIC ADP010760, DTIC, Fort Belvoir.

[2] Wood, R.J. (2008) The First Takeoff of a Biologically-Inspired At-Scale Robotic Insect. IEEE Transactions on Robotics, 24, 341-347.

https://doi.org/10.1109/TRO.2008.916997 
[3] Oyama, A. and Fuji, K. (2006) A Study on Airfoil Design for Future Mars Airplane. AIAA Paper 2006-1484, AIAA, Reston. https://doi.org/10.2514/6.2006-1484

[4] Braun, R.D., Wright, H.S., Croom, M.A., Levine, J.S. and Spencer, D.A. (2006) Design of the ARES Mars Airplane and Mission Architecture. Journal of Spacecraft and Rockets, 43, 1026-1034. https://doi.org/10.2514/1.17956

[5] Aono, H., Nonomura, T., Anyoji, M., Oyama, A. and Fujii, K. (2012) A Numerical Study of the Effects of Airfoil Shape on Low Reynolds Number Aerodynamics. Proceedings of the 8th International Conference on Engineering Computational Technology, Dubrovnik, 4-7 September 2012, 131.

[6] Anyoji, M., Nonomura, T., Aono, H., Oyama, A., Fujii, K., Nagai, H. and Asai, K. (2014) Computational and Experimental Analysis of a High-Performance Airfoil under Low-Reynolds-Number Flow Condition. Journal of Aircraft, 51, 1864-1872. https://doi.org/10.2514/1.C032553

[7] Mueller, T.J. and Delaurier, J.D. (2003) Aerodynamics of Small Vehicles. Annual Review of Fluid Mechanics, 35, 89-111.

https://doi.org/10.1146/annurev.fluid.35.101101.161102

[8] Mcmasters, J.H. and Henderson, M.L. (1980) Low-Speed Single-Element Airfoil Synthesis. Technical Soaring, 6, 1-21.

[9] Lissaman, P.B.S. (1983) Low-Reynolds-Number Airfoils. Annual Review of Fluid Mechanics, 15, 223-239. https://doi.org/10.1146/annurev.fl.15.010183.001255

[10] Schmitz, F.W. (1967) Aerodynamics of the Model Airplane: Part 1. Airfoil Measurements. NASA-TM-X-60976.

[11] Schmitz, F.W. (1980) The Aerodynamics of Small Reynolds Numbers. NASA TM-75816, NASA, Washington DC.

[12] Tsuchiya, T., Numata, D., Suwa, T. and Asai, K. (2013) Influence of Turbulence Intensity on Aerodynamic Characteristics of an NACA 0012 at Low Reynolds Numbers. AIAA Paper 2013-0065, AIAA, Reston. https://doi.org/10.2514/6.2013-65

[13] Wang, S., Zohn, Y., Alam, M.M. and Yang, H. (2014) Turbulent Intensity and Reynolds Number Effects on an Airfoil at Low Reynolds Numbers. Physics of Fluids, 26, Article ID: 115107. https://doi.org/10.1063/1.4901969

[14] Hoffmann, J.A. (1991) Effects of Freestream Turbulence on the Performance Characteristics of an Airfoil. AIAA Journal, 29, 1353-1354.

https://doi.org/10.2514/3.10745

[15] Simoni, D., Lengani, D., Ubaldi, M., Zunino, P. and Dellacasagrande, M. (2017) Inspection of the Dynamic Properties of Laminar Separation Bubbles: Free-Stream Turbulence Intensity Effects for Different Reynolds Numbers. Experiments in Fluids 58, Article No.66. https://doi.org/10.1007/s00348-017-2353-7

[16] Li, H. and Yang, Z. (2016) Numerical Study of Separated Boundary Layer Transition under Pressure Gradient. Proceedings of the 12 th International Conference on Heat Transfer, Fluid Mechanics and Thermodynamics, Malaga, 11-13 July 2016, 1759-1764.

[17] Balzer, W. and Fasel, H.F. (2016) Numerical Investigation of the Role of Free-Stream Turbulence in Boundary-Layer Separation. Journal of Fluid Mechanics, 801, 289-321. https://doi.org/10.1017/jfm.2016.424

[18] Abdalla, I.E. and Yang, Z. (2004) Numerical Study of the Instability Mechanism in Transitional Separating-Reattaching Flow. International Journal of Heat and Fluid Flow, 25, 593-605. https://doi.org/10.1016/j.ijheatfluidflow.2004.01.004

[19] Yang, Z. and Voke, P.R. (2001) Large-Eddy Simulation of Boundary Layer Separa- 
tion and Transition at a Change of Surface Curvature. Journal of Fluid Mechanics, 439, 305-333. https://doi.org/10.1017/S0022112001004633

[20] Yang, Z. and Abdalla, I.E. (2009) Effects of Free-Stream Turbulence on a Transitional Separated-Reattached Flow over a Flat Plate with a Sharp Leading Edge. International Journal of Heat and Fluid Flow, 30, 1026-1035. https://doi.org/10.1016/j.ijheatfluidflow.2009.04.010

[21] Langari, M. and Yang, Z. (2013) Numerical Study of the Primary Instability in a Separated Boundary Layer Transition under Elevated Free-Stream Turbulence. Physics of Fluids, 25, Article ID: 074106. https://doi.org/10.1063/1.4816291

[22] Lee, D., Kawai, S., Nonomura, T., Anyoji, M., Aono, H., Oyama, A., Asai, K. and Fuji, K. (2015) Mechanisms of Surface Pressure Distribution Within a Laminar Separation Bubble at Different Reynolds Numbers. Physics of Fluids, 27, Article ID: 023602. https://doi.org/10.1063/1.4913500

[23] Burns, T.F. and Mueller, T.J. (1982) Experimental Studies of the Eppler 61 Airfoil at Low Reynolds Numbers. AIAA Paper 1982-345, AIAA, Reston.

https://doi.org/10.2514/6.1982-345

[24] Tsuji, H. (1959) A Document on Turbulent Grid Design. Aeronautical Research Institute, University of Tokyo, Tokyo, 179-184. (In Japanese)

[25] Anyoji, M., Nose, K., Ida, S., Numata, D., Nagai, H. and Asai, K. (2011) Aerodynamic Measurements in the Mars Wind Tunnel at Tohoku University. AIAA Paper 2011-0852, AIAA, Reston. https://doi.org/10.2514/6.2011-852

[26] Sasaki, K. and Kiya, M. (1991) Three-Dimensional Vortex Structure in a Leading-Edge Separation Bubble at Moderate Reynolds Number. Journal of Fluids Engineering, 113, 405-410. https://doi.org/10.1115/1.2909510

[27] Rinoie, K. (2003) Laminar Separation Bubbles Formed on Airfoils. Journal of Japan Society Fluid Mechanics NAGARE, 22, 15-22. (In Japanese)

[28] Watmuff, J.H. (1999) Evolution of a Wave Packet into Vortex Loops in a Laminar Separation Bubble. Journal of Fluid Mechanics, 397, 119-169.

https://doi.org/10.1017/S0022112099006138

[29] Bruun, H.H. (1995) Hot-Wire Anemometry: Principles and Signal Analysis. Oxford University Press, Oxford. https://doi.org/10.1088/0957-0233/7/10/024

[30] Durst, F., Zanoun, E.S. and Pashtrapanska, M. (2001) in Situ Calibration of Hot Wires Close to Highly Heat-Conducting Walls. Experiments in Fluids, 31, 103-110. https://doi.org/10.1007/s003480000264

[31] Abdalla, I.E. and Yang, Z. (2005) Numerical Study of a Separated-Reattached Flow on a Blunt Plate. AIAA Journal, 43, 2465-2474. https://doi.org/10.2514/1.1317

[32] Kiya, M. and Sasaki, K. (1983) Structure of a Turbulent Separation Bubble. Journal of Fluid Mechanics, 137, 83-113. https://doi.org/10.1017/S002211208300230X

[33] Cherry, N.J., Hillier, R. and Latour, M.E.M.P. (1984) Unsteady Measurements in a Separated and Reattaching Flow. Journal of Fluid Mechanics, 144, 13-46. https://doi.org/10.1017/S002211208400149X

[34] Chandrasekhar, S. (1961) Hydrodynamic and Hydromagnetic Stability. Clarendon Press, Oxford.

[35] Tani, I. (1964) Low Speed Flows Involving Bubble Separations. Progress in Aerospace Sciences, 5, 70-103. https://doi.org/10.1016/0376-0421(64)90004-1

[36] O’Meara, M.M. and Mueller, T.J. (1987) Laminar Separation Bubble Characteristics on an Airfoil at Low Reynolds Number. AIAA Journal, 25, 1033-1041. 
https://doi.org/10.2514/3.9739

[37] Gerakopulos, R., Boutilier, M. and Yarusevych, S. (2010) Aerodynamic Characterization of a NACA 0018 Airfoil at Low Reynolds Numbers. AIAA Paper 2010-4629, AIAA, Reston. https://doi.org/10.2514/6.2010-4629

[38] Garmann, D.J. and Visbal, M.R. (2014) Dynamics of Revolving Wings for Various Aspect Ratios. Journal of Fluid Mechanics, 748, 932-956.

https://doi.org/10.1017/jfm.2014.212 


\section{Nomenclature}

\begin{tabular}{|c|c|}
\hline$C$ & Wave speed \\
\hline$c$ & Chord length \\
\hline$C_{D}$ & Drag coefficient \\
\hline$C_{L}$ & Lift coefficient \\
\hline$C_{m}$ & Pitching moment coefficients \\
\hline$C_{p}$ & Pressure coefficients \\
\hline$d$ & Wire diameter \\
\hline$f$ & Frequency \\
\hline $\bar{f}$ & Average characteristic frequency \\
\hline$H$ & Plate thickness \\
\hline$h$ & Shear layer thickness \\
\hline$K$ & Wave number \\
\hline$L_{u}$ & Streamwise integral length scale \\
\hline$M$ & Mesh size \\
\hline$P$ & Power spectrum density \\
\hline$T_{i}$ & Mainstream turbulence intensity \\
\hline $\operatorname{Re}_{c}$ & Chord length based Reynolds number \\
\hline $\operatorname{Re}_{H}$ & Thickness based Reynolds number \\
\hline$R_{u}$ & autocorrelation function \\
\hline$S_{F}$ & Solidity factor \\
\hline $\bar{U}$ & Local mean velocity in $x$ direction \\
\hline$U_{r m s}^{\prime}$ & $\begin{array}{l}\text { Root mean square value of fluctuating component of local velocity in } x \\
\text { direction }\end{array}$ \\
\hline$U_{\infty}$ & Mean streamwise velocity \\
\hline$u_{r m s}^{\prime}$ & $\begin{array}{l}\text { Root mean square value of fluctuating component of instantaneous } \\
\text { streamwise velocity }\end{array}$ \\
\hline $\bar{V}$ & Local mean velocity in $y$ direction \\
\hline$V_{r m s}^{\prime}$ & $\begin{array}{l}\text { Root mean square value of fluctuating component of local velocity in } y \\
\text { direction }\end{array}$ \\
\hline$v_{r m s}^{\prime}$ & $\begin{array}{l}\text { Root mean square value of fluctuating component of lateral instanta- } \\
\text { neous velocity }\end{array}$ \\
\hline$x$ & Horizontal coordinate \\
\hline$y$ & Vertical coordinate \\
\hline$\alpha$ & Angle of attack \\
\hline$\tau$ & Time difference \\
\hline
\end{tabular}

